\title{
SPECTRAL CONDITIONS AND AN EXPLICIT EXPRESSION FOR THE STABILIZATION OF HYBRID SYSTEMS IN THE PRESENCE OF FEEDBACK DELAYS
}

\author{
BY \\ K. L. COOKE (Pomona College, Claremont, California) \\ J. TURI (University of Texas at Dallas, Dallas, Texas) \\ AND \\ G. TURNER (Claremont Graduate School, Claremont, California)
}

\begin{abstract}
We consider the effect of feedback delays in the stabilization of linear time-invariant plants with sampled outputs. In particular, we obtain an estimate on the "minimum" sampling frequency (in terms of the spectrum of the plant and the delay in the feedback mechanism) needed for stabilization and provide an explicit expression for a stabilizing feedback control.
\end{abstract}

1. Introduction. Hybrid systems (i.e., continuous plant with discrete (sampled) controller) and their analysis have received considerable attention in the literature (see, e.g., [3], [5] and the references therein). Our main interest here is to study the effect of feedback delays in the stabilization of such systems. Throughout the paper we shall assume that the plant (to be stabilized) is linear time invariant and finite dimensional and that in the feedback process there is a time delay, $r$, which is greater or equal to some given constant (minimum delay), $r_{\min }>0$. Another parameter in the problem is the sampling period, $h$, and it is assumed that

$$
r=N h \geq r_{\min }, \quad N \in Z^{+},
$$

i.e., the delay is an integer multiple of the sampling period.

Consider the equation

$$
\dot{x}(t)=A x(t)+B u(t)
$$

where $A, B$ are $n \times n, n \times m$ matrices, respectively, and $x \in \mathbb{R}^{n}$ and $u \in \mathbb{R}^{m}$. For given minimum feedback delay, $r_{\min }>0$, we want to find the "maximum" sampling

Received May 30, 1991.

1991 Mathematics Subject Classification. Primary 93, 15, 34.

Parts of this research were carried out by the authors who were visitors at the Institute for Mathematics and its Applications [IMA] and was supported by IMA with funds provided by the National Science Foundation. Parts of this research have been excerpted from the third author's Ph.D. dissertation at the Claremont Graduate School (completed May 1991).

(C)1993 Brown University 
period, $h$ (or minimum sampling frequency $1 / h$ ), and the corresponding feedback control

$$
u(t)=K x([t / h] h-N h)
$$

such that $u(\cdot)$ given by (1.3) stabilizes the system (1.2), i.e., the zero solution of the equation

$$
\dot{x}(t)=A x(t)+B K x([t / h] h-N h)
$$

is exponentially stable. (Note that the symbol [.], appearing in (1.3) and (1.4), stands for the greatest integer part of the argument.) Equation (1.3) is "zero order hold," and together (1.2) and (1.3) constitute the hybrid system.

For $t \in[k h,(k+1) h), k \in Z^{+}$, the solution of Eq. (1.4) can be written as

$$
x(t)=e^{A(t-k h)} x(k h)+\int_{0}^{t-k h} e^{A \tau} d \tau B K x((k-N) h),
$$

and therefore (by continuity)

$$
x((k+1) h)=e^{A h} x(k h)+\int_{0}^{h} e^{A \tau} d \tau B K x((k-N) h) .
$$

Introducing the notations

$$
A_{D}:=e^{A h}, \quad B_{D}:=\int_{0}^{h} e^{A \tau} d \tau B
$$

and

$$
\xi_{k}=x(k h), \quad k=-N,-(N-1), \ldots, 0,1,2, \ldots,
$$

we obtain from (1.6) the difference equation

$$
\xi_{k+1}=A_{D} \xi_{k}+B_{D} K \xi_{k-N}, \quad k \geq 0 .
$$

We remark here that (1.9) represents a discrete-time version of the hybrid system (1.2) with

$$
u_{k}=u(k h)=K \xi_{k-N}
$$

and it is stabilizable whenever (1.2) is stabilizable assuming that the continuous plant, $A$, satisfies certain mild conditions. Also, as a simple consequence of $(1.5)$ and the fact that $e^{A(t-k h)}$ and $\int_{0}^{t-k h} e^{A \tau} d \tau$ are bounded functions of $t$ on $[k h,(k+1) h)$, if for some $K$ the sequence $\left\{\xi_{k}\right\}$ is exponentially convergent to zero, so is $x(t)$, the solution to (1.5).

In the next section we provide a sufficient condition for the stabilizability of (1.2), (1.3) and obtain an explicit expression for $K$ that allows an estimate on the maximum achievable sampling period.

2. Explicit and sufficient criteria for stabilization. The retarded equation

$$
\dot{x}=A x+B x([t-a]),
$$

where $a$ is a nonnegative integer and $A$ and $B$ are $n \times n$, has been studied by Cooke and Wiener [2]. 

then

If $x_{n}(t)$ is a solution of $(2.1)$ on $t \in[n, n+1)$ and we define $x(n-a)=c_{n-a}$,

$$
\dot{x}_{n}(t)=A x_{n}(t)+B c_{n-a} .
$$

If $A^{-1}$ exists and we let $x(n-i)=c_{n-i}, i=0,1,2, \ldots, n$, then the general solution of $(2.2)$ is

$$
x_{n}(t)=e^{A(t-n)} c-A^{-1} B c_{n-a}
$$

where $c$ is a constant of integration. Since $x_{n}(n)=c_{n}$, it follows from (2.3) that

$$
\begin{gathered}
c=c_{n}+A^{-1} B c_{n-a}, \\
x_{n}(t)=e^{A(t-n)} c_{n}+\left(e^{A(t-n)}-I\right) A^{-1} B c_{n-a},
\end{gathered}
$$

and

$$
x_{n-1}(t)=e^{A(t-n+1)} c_{n-1}+\left(e^{A(t-n+1)}-I\right) A^{-1} B c_{n-1-a} .
$$

Continuity requires $x_{n-1}(n)=x_{n}(n)=c_{n}$, so that from (2.5) and (2.6) we get

$$
c_{n}=e^{A} c_{n-1}-\left(e^{A}-I\right) A^{-1} B c_{n-(a+1)} .
$$

Looking for a nonzero solution $c_{n}=\lambda^{n} k$, with constant vector $k$, we conclude that $\lambda$ satisfies

$$
\operatorname{det}\left(\lambda^{a+1} I-e^{A} \lambda^{a}-\left(e^{A}-I\right) A^{-1} B\right)=0 .
$$

The asymptotic stability of the zero solution $x=0$ of $(2.1)$ is assured when all solutions $\lambda \in \mathbb{C}$ of the characteristic equation (2.8) have moduli less than 1 .

Equation (2.1) is very closely related to Eq. (1.4), and it is clear that the derivation of (1.6) and (2.6) are very similar.

The characteristic equation of (1.4), analogous to (2.8), is

$$
\operatorname{det}\left(\lambda^{N+1} I-A_{D} \lambda^{N}-B_{D} K\right)=0
$$

or, when $A^{-1}$ exists,

$$
\operatorname{det}\left(\lambda^{N+1} I-e^{A h} \lambda^{N}-\left(e^{A h}-I\right) A^{-1} B K\right)=0 .
$$

Conditions sufficient to ensure that the zero solution of (1.4) is asymptotically stable guarantee the stabilizability of the hybrid equation (1.2), (1.3).

When $A$ and $B$ are $n \times n$ and nonsingular, $K$ can be specified as a function of $A$, the spectrum of $A$, and the sampling period $h$ so that $x=0$ is an asymptotically stable solution of (1.4). From this, bounds on the spectrum of $A$ and on the maximum sampling period $h$ can be obtained ensuring the stabilization of (1.2), (1.3). In particular, we have the following result.

THEOREM 2.1. Let $\alpha_{0}$ be the double (real) zero of the polynomial

$$
p(\alpha)=\alpha^{N+1}-\alpha^{N}+\frac{N^{N}}{(N+1)^{N+1}},
$$

so that for small $\varepsilon>0, \alpha_{0}-\delta_{2}$ and $\alpha_{0}+\delta_{1}$ are the real zeros of the polynomial

$$
p_{\varepsilon}(\alpha)=\alpha^{N+1}-\alpha^{N}+\frac{N^{N}}{(N+1)^{N+1}}-\varepsilon .
$$


Let $r=N h, N \in Z^{+}, h \in(0, r]$, and let $A$ and $B$ be $n \times n$ and nonsingular.

Then the closed-loop hybrid feedback system (1.2), (1.3) is stabilizable if

$$
\operatorname{Re} \sigma(A) \subset\left(-\infty, \frac{1}{h} \ln \frac{r+h}{r}\right) .
$$

Any matrix of the form

$$
K=\left(\varepsilon-\frac{N^{N}}{(N+1)^{N+1}}\right) B^{-1} A\left(e^{A h}-I\right)^{-1} e^{(N+1) A h}
$$

is a stabilizing matrix if $\varepsilon$ is determined so that

$$
\delta_{1}<e^{-\max \operatorname{Re} \sigma(A h)}-\frac{N}{N+1} .
$$

The following two lemmas play an important role in the proof of Theorem 2.1.

Lemma 2.2. Suppose $p(z)=z^{N+1}-z^{N}+N^{N} /(N+1)^{N+1}, N \in Z^{+}$. Then if $\alpha$ is a zero of $p(z),|\alpha| \leq N /(N+1)$.

Proof. Elementary calculations show that

$$
p(z)=z^{N+1}-z^{N}+\frac{N^{N}}{(N+1)^{N+1}}=\left(z-\frac{N}{N+1}\right)^{2}\left(\sum_{j=1}^{N} \frac{j N^{N-j-1}}{(N+1)^{N-j}} z^{j-1}\right) .
$$

Introducing the notation $g(z)=\sum_{i=0}^{N-1} a_{i} z^{i}$ for the second term on the right-hand side of (2.11), we can observe that the $a_{i}$ 's satisfy

(i) $a_{N-1} \geq a_{N-2} \geq \cdots \geq a_{0} \geq 0$

(ii) for $i \in[1, N-1], a_{i-1} / a_{i}=(i /(i+1))(N /(N+1))<N /(N+1)$.

An application of the Eneström-Kakeya Theorem [7, p. 173] shows that if $\beta$ is a zero of $g(z)$ then $|\beta|<N /(N+1)$. It follows that if $\alpha$ is a zero of $p(z)$ then $|\alpha| \leq N /(N+1)$.

Lemma 2.3. If the $n \times n$ matrix $A$ is nonsingular and $a_{i} \neq a_{j}, 1 \leq i<j \leq m$, $a_{i} \in \mathbb{C}$, then the $m n \times m n$ block Vandermonde matrix

$$
S=\left[\begin{array}{cccc}
I_{n} & I_{n} & \ldots & I_{n} \\
a_{1} A & a_{2} A & \ldots & a_{m} A \\
a_{1}^{2} A^{2} & a_{2}^{2} A^{2} & \ldots & a_{m}^{2} A^{2} \\
\vdots & \vdots & & \vdots \\
a_{1}^{m-1} A^{m-1} & a_{2}^{m-1} A^{m-1} & \ldots & a_{m}^{m-1} A^{m-1}
\end{array}\right]
$$

is nonsingular.

Proof. Straightforward calculations show that $\operatorname{det} S=$

$$
\left|\begin{array}{ccccc}
I_{n} & I_{n} & I_{n} & \ldots & I_{n} \\
0 & \left(a_{2}-a_{1}\right) A & \left(a_{3}-a_{1}\right) A & \ldots & \left(a_{m}-a_{1}\right) A \\
0 & \left(a_{2}^{2}-a_{2} a_{1}\right) A^{2} & \left(a_{3}^{2}-a_{3} a_{1}\right) A^{2} & \ldots & \left(a_{m}^{2}-a_{m} a_{1}\right) A^{2} \\
\vdots & \vdots & \vdots & & \vdots \\
0 & \left(a_{2}^{m-1}-a_{2}^{m-2} a_{1}\right) A^{m-1} & \left(a_{3}^{m-1}-a_{3}^{m-2} a_{1}\right) A^{m-1} & \ldots & \left(a_{m}^{m-1}-a_{m}^{m-2} a_{1}\right) A^{m-1}
\end{array}\right| .
$$


(If $R_{i}$ is the $i$ th row of block matrices of (2.5) then the above equality may be seen by placing $\left(-a_{i} A\right) R_{i}+R_{i+1}$ into $R_{i+1}$ for $i=m-1, m-2, \ldots, 1$ respectively.)

We then obtain

$$
\begin{aligned}
& \operatorname{det} S=\left|\begin{array}{cccc}
\left(a_{2}-a_{1}\right) A & \left(a_{3}-a_{1}\right) A & \ldots & \left(a_{m}-a_{1}\right) A \\
\left(a_{2}^{2}-a_{2} a_{1}\right) A^{2} & \left(a_{3}^{2}-a_{3} a_{1}\right) A^{2} & \ldots & \left(a_{m}^{2}-a_{m} a_{1}\right) A^{2} \\
\vdots & \vdots & & \vdots \\
\left(a_{2}^{m-1}-a_{2}^{m-2} a_{1}\right) A^{m-1} & \left(a_{3}^{m-1}-a_{3}^{m-2} a_{1}\right) A^{m-1} & \ldots & \left(a_{m}^{m-1}-a_{m}^{m-2} a_{1}\right) A^{m-1}
\end{array}\right| \\
& =\prod_{j=2}^{m}\left(a_{j}-a_{1}\right)^{(m-1) n}\left|\begin{array}{cccc}
A & A & \ldots & A \\
a_{2} A^{2} & a_{3} A^{2} & \ldots & a_{m} A^{2} \\
a_{2}^{2} A^{3} & a_{3}^{2} A^{3} & \ldots & a_{m}^{2} A^{3} \\
\vdots & \vdots & & \vdots \\
a_{2}^{m-2} A^{m-1} & a_{3}^{m-2} A^{m-1} & \ldots & a_{m}^{m-2} A^{m-1}
\end{array}\right| \\
& =\prod_{j=2}^{m}\left(a_{j}-a_{1}\right)^{(m-1) n}\left|\begin{array}{cccc}
A & A & \ldots & A \\
0 & \left(a_{3}-a_{2}\right) A^{2} & \ldots & \left(a_{m}-a_{2}\right) A^{2} \\
0 & \left(a_{3}^{2}-a_{3} a_{2}\right) A^{3} & \ldots & \left(a_{m}^{2}-a_{m} a_{2}\right) A^{3} \\
\vdots & \vdots & & \vdots \\
0 & \left(a_{3}^{m-2}-a_{3}^{m-3} a_{2}\right) A^{m-1} & \ldots & \left(a_{m}^{m-2}-a_{m}^{m-3} a_{2}\right) A^{m-1}
\end{array}\right| \\
& =\prod_{j=2}^{m}\left(a_{j}-a_{1}\right)^{(m-1) n}(\operatorname{det} A)\left|\begin{array}{ccc}
\left(a_{3}-a_{2}\right) A^{2} & \ldots & \left(a_{m}-a_{2}\right) A^{2} \\
\left(a_{3}^{2}-a_{3} a_{2}\right) A^{3} & \ldots & \left(a_{m}^{2}-a_{m} a_{2}\right) A^{3} \\
\vdots & & \vdots \\
\left(a_{3}^{m-2}-a_{3}^{m-3} a_{2}\right) A^{m-1} & \ldots & \left(a_{m}^{m-2}-a_{m}^{m-3} a_{2}\right) A^{m-1}
\end{array}\right| \\
& =\left(\prod_{j=2}^{m}\left(a_{j}-a_{1}\right)^{(m-1) n}\right)\left(\prod_{k=3}^{m}\left(a_{k}-a_{2}\right)^{(m-2) n}\right) \operatorname{det} A \\
& \left|\begin{array}{cccc}
A^{2} & A^{2} & \ldots & A^{2} \\
a_{3} A^{3} & a_{4} A^{3} & \ldots & a_{m} A^{3} \\
\vdots & \vdots & & \vdots \\
a_{3}^{m-3} A^{m-1} & a_{4}^{m-3} A^{m-1} & \ldots & a_{m}^{m-3} A^{m-1}
\end{array}\right| \\
& =\left(\prod_{k_{1}=2}^{m}\left(a_{k_{1}}-a_{1}\right)^{(m-1) n}\right)\left(\prod_{k_{2}=3}^{m}\left(a_{k_{2}}-a_{2}\right)^{(m-2) n}\right)\left(\prod_{k_{3}=4}^{m}\left(a_{k_{3}}-a_{3}\right)^{(m-3) n}\right) \\
& \cdot(\operatorname{det} A)\left(\operatorname{det} A^{2}\right)\left|\begin{array}{ccc}
A^{3} & \ldots & A^{3} \\
a_{4} A^{4} & \cdots & a_{m} A^{4} \\
\vdots & & \vdots \\
a_{4}^{m-4} A^{m-1} & \ldots & a_{m}^{m-4} A^{m-1}
\end{array}\right|
\end{aligned}
$$




$$
\begin{aligned}
= & \left(\prod_{k_{1}=2}^{m}\left(a_{k_{1}}-a_{1}\right)^{(m-1) n}\right)\left(\prod_{k_{2}=3}^{m}\left(a_{k_{2}}-a_{2}\right)^{(m-2) n}\right) \\
& \ldots\left(\prod_{k_{m-2}=m-1}^{m}\left(a_{k_{m-2}}-a_{m-2}\right)^{2 n}\right) \\
= & \left.\prod_{i=1}^{m-1}\left(\prod_{k_{i}=i+1}^{m}\left(a_{k_{i}}-a_{i}\right)^{(m-i) n}\right)^{2} \cdot \operatorname{det}\left(A A^{2} \cdots A^{m-1}\right) \cdot \mid A_{m-1} A^{m-1}\right) \\
= & \prod_{i=1}^{m-1}\left(\prod_{k_{i}=i+1}^{m}\left(a_{k_{i}}-a_{i}\right)^{(m-i) n} A_{m}^{m-2} A^{m-1} \mid\right.
\end{aligned}
$$

The right-hand side of (2.13) is zero if and only if $a_{k_{i}}=a_{i}, 1 \leq i<k_{i} \leq m$ or $\operatorname{det} A=0$. By hypothesis, then, $S$ is nonsingular.

Now we are ready to prove our main result.

Proof of Theorem 2.1. Introduce the notation

$$
L_{\varepsilon}(\lambda)=\lambda^{N+1} I-e^{A h} \lambda^{N}-\left(e^{A h}-I\right) A^{-1} B K
$$

where

$$
K=\left(\varepsilon-\frac{N^{N}}{(N+1)^{N+1}}\right) B^{-1} A\left(e^{A h}-I\right)^{-1} e^{(N+1) A h} .
$$

It is understood that $x=0$ is an asymptotically stable solution of $(1.2),(1.3)$ if, for all $\lambda$ satisfying $\operatorname{det} L_{\varepsilon}(\lambda)=0$, we have $|\lambda|<1 . L_{\varepsilon}(\lambda)$ is a monic matrix polynomial of degree $N+1$ and we wish to capture spectral information, i.e., determine the latent roots (see [6]) of $L_{\varepsilon}(\lambda)$.

Temporarily, we shall assume $\varepsilon=0$ and use the simplified notation $L_{0}(\lambda)=L(\lambda)$. Note that $L\left(\alpha e^{A h}\right)=\left(\alpha^{N+1}-\alpha^{N}+N^{N} /(N+1)^{N+1}\right) e^{(N+1) A h}$ and this equals 0 if and only if $p_{0}(\alpha)=p(\alpha)=\alpha^{N+1}-\alpha^{N}+N^{N} /(N+1)^{N+1}=0$.

Now $p^{\prime}(\alpha)=\alpha^{N-1}((N+1) \alpha-N)$, so $p^{\prime}(\alpha)=0$ iff $\alpha=0$ or $N /(N+1)$. Since $p(0)$ is not 0 but $p(N /(N+1))=0$, it follows that $\alpha=N /(N+1)$ is the only zero of $p(\alpha)$ of multiplicity $>1$. In fact it is a zero of multiplicity 2 and we may write

$$
p(\alpha)=\left(\alpha-\frac{N}{N+1}\right)^{2}\left(\sum_{j=1}^{N} \frac{j N^{N-j-1}}{(N+1)^{N-j}} \alpha^{j-1}\right)
$$

where the zeros of

$$
g(\alpha)=\sum_{j=1}^{N} \frac{j N^{N-j-1}}{(N+1)^{N-j}} \alpha^{j-1}
$$

are necessarily distinct. 
Now, we may conclude from Lemma 2.3, that if all zeros of $p(\alpha)$ are distinct, then the $(N+1) n \times(N+1) n$ block Vandermonde matrix associated with the $N+1$ solvents, $\left\{\alpha_{i} e^{A h}\right\}_{i=1}^{N+1}$, of $L(\lambda)$

$$
\left[\begin{array}{cccc}
I_{n} & I_{n} & \ldots & I_{n} \\
\alpha_{1} e^{A h} & a_{2} e^{A h} & \ldots & \alpha_{N+1} e^{A h} \\
\vdots & \vdots & & \vdots \\
\alpha_{1}^{N} e^{A h N} & \alpha_{2}^{N} e^{A h N} & \ldots & \alpha_{N+1}^{N} e^{A h N}
\end{array}\right]
$$

is nonsingular, in which case the set $\left\{\alpha_{i} e^{A h}\right\}_{i=1}^{N+1}$ is a complete set of solvents of $L(\lambda)$ (see, e.g., [6]). However, since $p(\alpha)$ has a double zero at $\alpha=N /(N+1)$, only (at most) $N$ of the set of $N+1$ zeros are distinct.

Next we consider the case $\varepsilon>0$, i.e., $L_{\varepsilon}(\lambda)$ and (respectively) $p_{\varepsilon}(\alpha)$.

The graphs of $p(\alpha)$ and $p_{\varepsilon}(\alpha)$ are shown in Figs. 1 and 2, respectively.

(Note. If $N$ is an even integer then the degree of $p(\alpha)$ or $p_{\varepsilon}(\alpha)$ is odd and, therefore, there exists a second or third real zero. However, this root, along with the other complex roots, has modulus less than $N /(N+1)$. For this reason we examine the graphs of $p(\alpha)$ and $p_{\varepsilon}(\alpha)$ near $\alpha=N /(N+1)$. We are interested in the increase of magnitude of the zero of maximum modulus of $p_{\varepsilon}(\alpha)$ with respect to the zero of maximum modulus of $p(\alpha)$.

Since the zeros of a polynomial are continuous functions of the coefficients [7],

$$
p_{\varepsilon}(\alpha)=\left(\alpha-\left(\frac{N}{N+1}+\delta_{1}\right)\right)\left(\alpha-\left(\frac{N}{N+1}-\delta_{2}\right)\right) \cdot \prod_{i=3}^{N+1}\left(\alpha-\hat{\alpha}_{i}\right)
$$

where $\left|\hat{\alpha}_{i}\right|=\left|\alpha_{i}\right| \pm \delta_{i}, 3 \leq i \leq N+1, \alpha_{i}$ is a zero of $g(\alpha)$ and the $\delta_{i}$ 's are continuous functions of $\varepsilon$. Then the zeros of $p_{\varepsilon}(\alpha)$ are $\left\{(N /(N+1))-\delta_{2},(N /(N+1))+\delta_{1}\right.$, $\left.\left\{\hat{\alpha}_{i}\right\}_{i=3}^{N+1}\right\}$ and are distinct if $\varepsilon$ is sufficiently small. It then follows that

$$
\left.\mathscr{S}=\left\{\left((N /(N+1))-\delta_{2}\right) e^{A h},\left((N /(N+1))+\delta_{1}\right) e^{A h},\left\{\hat{\alpha}_{i} e^{A h}\right\}\right\}_{i=3}^{N+1}\right\}
$$

is a complete set of solvents of $L_{\varepsilon}(\lambda)$.

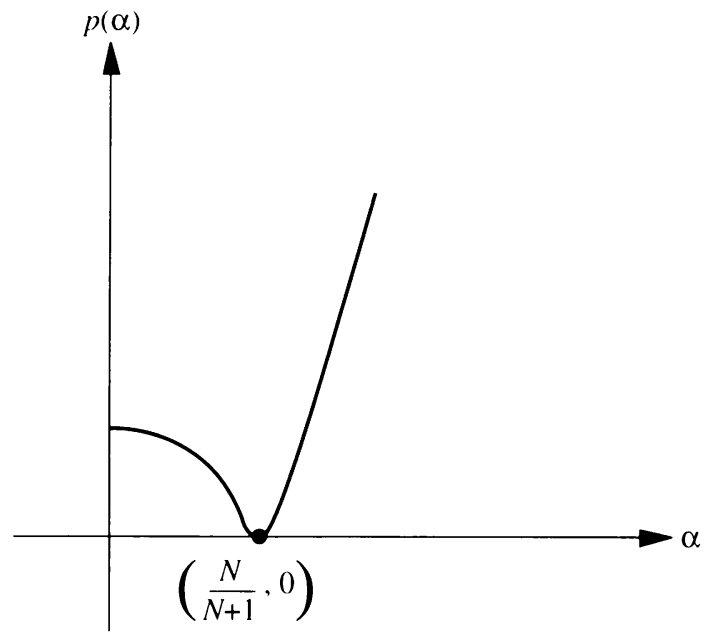

Fig. 1. Graph of $p(\alpha)$ 


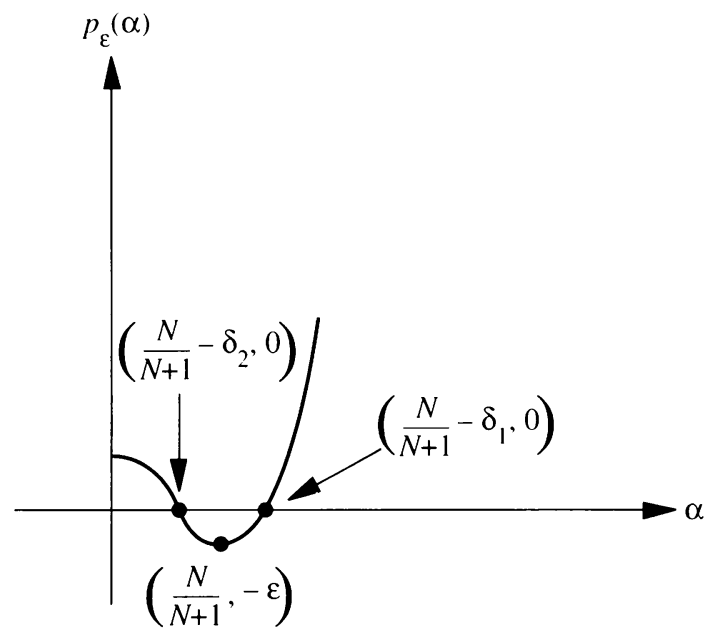

FIG. 2. Graph of $p_{\varepsilon}(\alpha)$

Now, from Lemma 2.2, if $\alpha$ is a zero of $p(\alpha)$ then $|\alpha| \leq N /(N+1)$ and, in particular, the zeros of $g(\alpha)=\sum_{j=1}^{N}\left(j N^{N-j-1} /(N+1)^{N-j}\right) \alpha^{j-1}$ all have modulus smaller than the real zero $(=N /(N+1))$. From the continuity of the zeros of $p(\alpha)$ [7], it follows that the zero of maximum modulus of $p_{\varepsilon}(\alpha)$ is $\alpha=(N /(N+1))+\delta_{1}$ (for $\varepsilon$ sufficiently small).

Moreover, if $\mathscr{S}$ is a complete set of solvents of $L_{\varepsilon}(\lambda)$, then the latent roots of $L_{\varepsilon}(\lambda)$ are $\lambda_{i}=\hat{\alpha}_{i} e^{\lambda_{A h}}$ where $\hat{\alpha}_{i}$ is a zero of $p_{\varepsilon}(\alpha)$ and $\lambda_{A h}$ is an eigenvalue of $A h$. Then,

$$
\begin{aligned}
\left|\lambda_{i}\right|=\left|\hat{\alpha}_{i}\right|\left|e^{\lambda_{A h}}\right|=\left|\hat{\alpha}_{i}\right| e^{\operatorname{Re} \lambda_{A h}} & \leq\left((N /(N+1))+\delta_{1}\right) e^{\operatorname{Re} \lambda_{A h}} \\
& =\left((N /(N+1))+\delta_{1}\right) e^{\operatorname{Re} h \lambda_{A}} \\
& \leq\left((N /(N+1))+\delta_{1}\right) e^{\max h \operatorname{Re} \lambda_{A}} \\
& =\left((N /(N+1))+\delta_{1}\right) e^{h \max \operatorname{Re} \lambda_{A}}
\end{aligned}
$$

where $\max \operatorname{Re} \lambda_{A}$ is the largest real part of all eigenvalues of $A$.

In view of inequality (2.20), the asymptotic stability of the zero solution of (1.4) is guaranteed (or (1.2), (1.3) is stabilized) if

$$
\left((N /(N+1))+\delta_{1}\right) e^{h \max \operatorname{Re} \lambda_{A}}<1
$$

is satisfied for some $\delta_{1}>0$, or equivalently, if we have

$$
e^{-h \max \operatorname{Re} \lambda_{A}}>N /(N+1) .
$$

Since $(N+1) / N=(r+h) / r$, it is easy to see that $(2.21)$ is equivalent to

$$
\operatorname{Re} \sigma(A) \subset\left(-\infty, \frac{1}{h} \ln \frac{r+h}{r}\right) .
$$


We may express this spectral-bound sufficiency condition in terms of $r$ and $N$, the number of sampling intervals. In this case,

$$
\frac{1}{h} \ln \frac{r+h}{r}=\frac{N}{r} \ln \frac{N+1}{N}
$$

with $h \in(0, r]$ so that the positive integer $N \in[1, \infty)$. This motivates the following Corollary 2.4. Let $h \in(0, r], N \in Z^{+}$, and $A$ and $B$ be $n \times n$ and nonsingular. Then $\operatorname{Re} \sigma(A) \subset(-\infty, 1 / r)$ implies that the system (1.2), (1.3) is stabilizable.

Proof. As the integer $N \rightarrow+\infty$ and $h \rightarrow 0^{+}, \lim (1 / h) \ln ((r+h) / r)=1 / r$. In fact, the function (of a real variable $x) \quad \varphi(x)=(1 / x) \ln ((r+x) / r)$ is monotone decreasing. So therefore when $h \in(0, r]$, it follows that $(1 / h) \ln ((r+h) / r) \in$ $[(1 / r) \ln 2,1 / r)$.

If $\max \operatorname{Re} \lambda_{A}<1 / r$, we can find $h \in(0, r]$ such that $(1 / h) \ln ((r+h) / r) \in$ $\left(\max \operatorname{Re} \lambda_{A}, 1 / r\right)$. Hence $\max \operatorname{Re} \lambda_{A}<(1 / h) \ln ((r+h) / r)$, which implies that (1.2), (1.3) is stabilizable.

RemarK 2.5. Assume that $\max \operatorname{Re} \lambda_{A}<1 / r$, and we wish to determine the minimum $N$ (or, equivalently, the largest $h$ ) such that (1.2), (1.3) is stabilizable; however, no explicit formulation for $h$ or $N$ as a function of $\max \operatorname{Re} \lambda_{A}$ and $r$ may be obtained, for this would require either

$$
\frac{1}{h} \ln \frac{r+h}{r}>\max \operatorname{Re} \lambda_{A}
$$

or

$$
\frac{N}{r} \ln \frac{N+1}{N}>\max \operatorname{Re} \lambda_{A} \text {. }
$$

To determine $h$ or $N$ in (2.15) or (2.16) (respectively), we must resort to numerical means.

EXAMPLE 2.6. Suppose we wish to stabilize

$$
\begin{aligned}
& \dot{x}=\left[\begin{array}{cc}
.28 & 10 \\
0 & -3
\end{array}\right] x+\left[\begin{array}{cc}
2 & -8 \\
0 & -.1
\end{array}\right] u, \\
& u=K x([t / h] h-3.0), \quad N h=3.0=r .
\end{aligned}
$$

First, we utilize the criterion described in the previous theorem to see if this system is stabilizable. If so, we will then determine the maximum sampling period $h$ and finally explicitly determine the stabilizing matrix $K$.

In this example, $r=3=N h$. Since $\max \operatorname{Re} \lambda_{A}=.28$ and this is $<1 / r=1 / 3$, we are assured this system is stabilizable (i.e., can determine $K$ to stabilize).

Next, we determine $h_{\max }$ for this to occur. We proceed, first determining (the smallest) $N$ such that $(N / r) \ln ((N+1) / N)=(N / 3) \ln ((N+1) / N)>.28$. In this case, $N=3$ suffices giving $h_{\max }=1$. Finally,

$$
\begin{aligned}
& K=\left(\varepsilon-\left(3^{3} / 4^{4}\right)\right)\left[\begin{array}{cc}
2 & -8 \\
0 & -.1
\end{array}\right]^{-1} \cdot\left[\begin{array}{cc}
.28 & 10 \\
0 & -3
\end{array}\right] \\
& \cdot\left(\exp \left(\left[\begin{array}{cc}
.28 & 10 \\
0 & -3
\end{array}\right]\right)-I\right) \exp \left(4\left[\begin{array}{cc}
.28 & 10 \\
0 & -3
\end{array}\right]\right)
\end{aligned}
$$


and it then remains to determine $\varepsilon$. We compute $e^{-\max \operatorname{Re} \sigma(A)}-N /(N+1)=e^{-.28}$ $.75 \approx .0058$. Now $p(\alpha)=\alpha^{4}-\alpha^{3}+3^{3} / 4^{4}=(\alpha-.75)^{2}\left(\alpha^{2}+(\alpha / 2)+3 / 16\right)$ and we want to select $\varepsilon$ so that $p_{\varepsilon}(\alpha)=\alpha^{4}-\alpha^{3}+3^{3} / 4^{4}-\varepsilon$ is such that the larger of the two real zeros belongs to $(.75, .7558)$. So select, for example, $\varepsilon=4.516 \times 10^{-6}$.

This last example suggests the following corollary that will explicitly formulate $\varepsilon$ as a function of $\max \operatorname{Re} \lambda_{A}$ and $N$.

REMARK 2.7. Assume the hypothesis of this section's theorem. Let

$\varepsilon=\left(\frac{1}{2}\left(\frac{N}{N+1}+e^{-\max \operatorname{Re} \sigma(A)}\right)\right)^{N+1}-\left(\frac{1}{2}\left(\frac{N}{N+1}+e^{-\max \operatorname{Re} \sigma(A)}\right)\right)^{N}+\frac{N^{N}}{(N+1)^{N+1}}$

Then $K=\left(\varepsilon-N^{N} /(N+1)^{N+1}\right) B^{-1} A\left(e^{A h}-I\right)^{-1} e^{(N+1) A}$ stabilizes (1.2), (1.3).

Proof. We want $\varepsilon$ such that $\delta_{1}<e^{-\max \operatorname{Re}(A)}-N /(N+1)$ where $p((N /(N+1))+$ $\left.\delta_{1}\right)=0$; that is, the larger of the two (positive) real zeros of $p_{\varepsilon}(\alpha),(N /(N+1))+\delta_{1}$, must be in the interval $\left(N /(N+1),(N /(N+1))+\left(e^{-\max \operatorname{Re} \sigma(A)}-N /(N+1)\right)\right)=$ $\left(N /(N+1), e^{-\max \operatorname{Re} \sigma(A)}\right)$.

Choose $\varepsilon$, for example, so that $(N /(N+1))+\delta_{1}$ is the midpoint of this interval, i.e., so that $(N /(N+1))+\delta_{1}=(1 / 2)\left(e^{-\max \operatorname{Re} \sigma(A)}+N /(N+1)\right)$. But since $(N /(N+1))+\delta_{1}$ is a zero of $p_{\varepsilon}(\alpha)$ it follows that $p_{\varepsilon}\left((1 / 2)\left(e^{-\max \operatorname{Re} \sigma(A)}+N /(N+1)\right)\right)=$ 0 . Equivalently,

$$
\begin{aligned}
\left(\frac{1}{2}\left(e^{-\max \operatorname{Re} \sigma(A)}+\frac{N}{N+1}\right)\right)^{N+1}-\left(\frac{1}{2}\left(e^{-\max \operatorname{Re} \sigma(A)}+\frac{N}{N+1}\right)\right)^{N} & \\
& +\frac{N^{N}}{(N+1)^{N+1}}-\varepsilon=0 .
\end{aligned}
$$

Solving for $\varepsilon$ produces the result.

3. Conditions for oscillatory solutions. We now consider oscillatory solutions of the hybrid system (1.2), (1.3).

A scalar solution, $x(t)$, of a differential equation is said to oscillate if it has arbitrarily large zeros; i.e., for any $t_{2}>t_{1}$, there exists a point $t_{3}>t_{2}$ such that $x\left(t_{3}\right)=0$. The solution of a system of differential equations is said to oscillate if each component is oscillatory [4].

A necessary and sufficient condition for solutions of a system of differential equations to be oscillatory is that all solutions of the system's characteristic equation are nonreal. For analogous discrete and hybrid systems, oscillatory solutions occur when solutions of the characteristic equation are nonpositive [4].

Applying this criterion to the difference equation (1.9) of Sec. 1, we conclude that the system (1.2), (1.3) is oscillatory if and only if there exist no positive solutions $\lambda$ of the characteristic equation (2.10). 


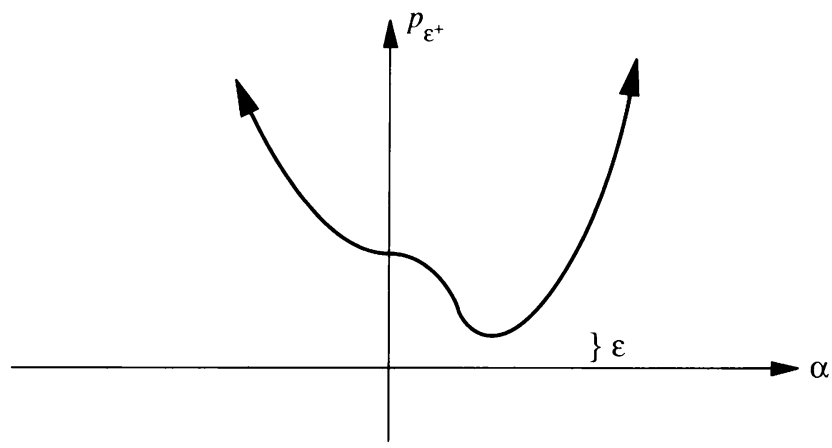

Fig. 3

Specifically, we now offer an explicit criterion concerning oscillatory solutions to (1.2), (1.3).

Theorem 3.1. Suppose $A$ is $n \times n$ with real eigenvalues, $B$ is $n \times n$ and nonsingular, and $\varepsilon$ is positive. Then (1.2), (1.3) is oscillatory with

$$
K=\left(-\varepsilon-N^{N} /(N+1)^{N+1}\right) B^{-1} A\left(e^{A h}-I\right)^{-1} e^{(N+1) A h} .
$$

Proof. Analogous to (2.14), (2.15), let

$$
L_{\varepsilon^{+}}(\lambda)=\lambda^{N+1} I-e^{A h} \lambda^{N}+\left(N^{N} /(N+1)^{N+1}+\varepsilon\right) e^{(N+1) A h} .
$$

Now, again considering $L(\lambda)(\varepsilon=0)$, we see that $\lambda=\alpha_{i} e^{A h}$ is a solvent of $L(\lambda)$ when $\alpha_{i}$ is a zero of

$$
p(\alpha)=\alpha^{N+1}-\alpha^{N}+N^{N} /(N+1)^{(N+1)}
$$

and $\hat{\alpha}_{i} e^{A h}$ is a solvent of $L_{\varepsilon^{+}}(\lambda)$ with

$$
p_{\varepsilon^{+}}(\alpha)=\alpha^{N+1}-\alpha^{N}+N^{N} /(N+1)^{N+1}+\varepsilon .
$$

The set $\left\{\hat{\alpha}_{i} e^{A h}\right\}_{i=1}^{N+1}$ is a complete set of solvents so the latent roots of $L_{\varepsilon^{+}}(\lambda)$ are $\lambda=\hat{\alpha}_{i} e^{\lambda_{A h}}$.

There are two cases to consider.

When $N$ is an odd integer, $p_{\varepsilon^{+}}(\alpha)$ looks like the graph in Fig. 3.

That is, all zeros of $p_{\varepsilon^{+}}(\alpha), \hat{\alpha}_{i}$, are of the form $x+i y, y \neq 0$. If $A$ has real eigenvalues then the imaginary part of each latent root of $L_{\varepsilon^{+}}(\lambda)$ is nonzero. In particular, the latent roots then are nonpositive.

If $N$ is an even integer then $p_{\varepsilon^{+}}(\alpha)$ looks like the graph in Fig. 4 (see p. 158).

The one real zero of $p_{\varepsilon^{+}}(\alpha)$ is negative, so then if the eigenvalues of $A$ are real, the latent roots of $L_{\varepsilon^{+}}(\lambda)$ are either complex (nonzero imaginary part as when $N$ was odd) or $<0$. Thus, in either case, the latent roots are nonpositive.

From the previous lemma, we conclude that every solution of $(1.2),(1.3)$ must be oscillatory (i.e., oscillates componentwise). 


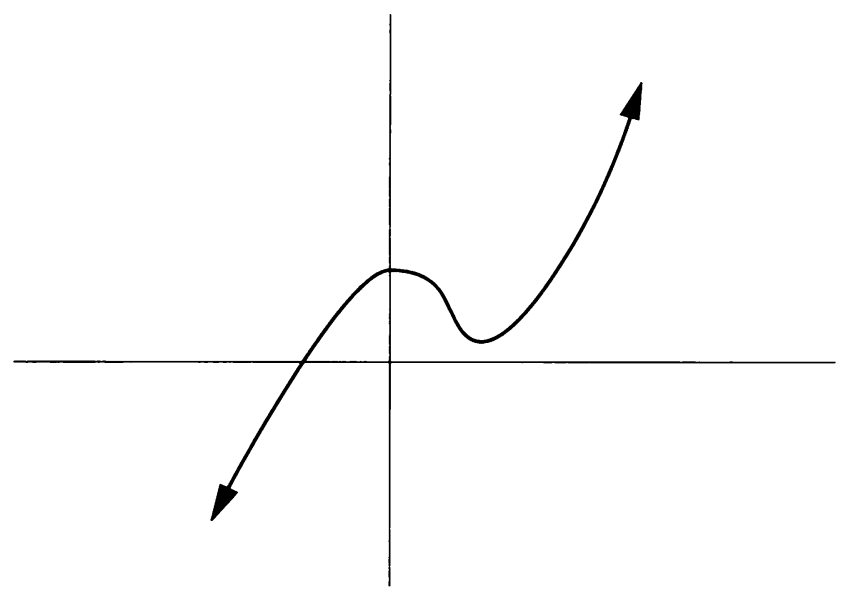

FIG. 4

4. Some generalizations. In [12] Yong considers the continuous system

$$
\dot{x}=A x+B u
$$

with feedback control

$$
u=K x(t-r),
$$

where $A$ is $n \times n, B$ is $n \times m$, and $r>0$. The system is defined to be $r$-stabilizable if there exists a matrix $K$ that stabilizes (4.1); that is, if $K$ ensures that the solution of

$$
\dot{x}=A x+B K x(t-r)
$$

is asymptotically stable for any initial condition. The system is defined to be uniformly $r$-stabilizable if there exists a matrix $K$ that ensures the asymptotic stability of

$$
\dot{x}=A x+B K x\left(t-r^{\prime}\right)
$$

for all $r^{\prime} \in[0, r]$.

It is shown that if $[A, B]$ is completely controllable and $\operatorname{Re} \sigma(A) \subset(-\infty, 0]$, then (4.3) is $r$-stabilizable. (4.3) is also $r$-stabilizable if $[A, B]$ is completely controllable, $\operatorname{Re} \sigma(A) \subset(-\infty, 0] \cup\{a\}$ and $a \in(0,1 / r)$ when the Jordan blocks of $A$ corresponding to $a$ are of order 1 . Similar conditions are also delineated that ensure the uniform $r$-stabilizability (discussed for single-input systems only) of (4.3).

In the case of the hybrid system (1.2), (1.3), Corollary 2.4 guarantees the $r$ stabilizability if $\operatorname{Re} \sigma(A) \subset(-\infty, 1 / r)$ and $A$ and $B$ are $n \times n$ and nonsingular. Note that for any $r^{\prime} \in(0, r], 1 / r^{\prime} \geq 1 / r$. It follows that if we assume $\operatorname{Re} \sigma(A)<1 / r$, then $\operatorname{Re} \sigma(A)<1 / r^{\prime}$ and (1.2), (1.3) is $r^{\prime}$-stabilizable. But the $K$ that stabilizes (1.2), (1.3) may not be sufficient to ensure the asymptotic stability of

$$
x=A x+B K\left([t / h] h-r^{\prime}\right) .
$$

In other words, the existence of sufficient spectral conditions on $A$ ensuring the uniform $r$-stabilizability of (1.2), (1.3) is not evident. 
Yong has also detailed conditions [13] sufficient for the stabilization of the pure discrete system

$$
\begin{aligned}
x(k+1) & =A x(k)+b u(k), \\
u(k) & =K x(k-N)
\end{aligned}
$$

where $N$ is a positive integer, $A$ is $n \times n, b$ is $n \times 1$, and $[A, b]$ is completely controllable.

If it is assumed, once again, that $A$ and $B$ are both $n \times n$ and nonsingular, we may use an argument analogous to Theorem 2.1 to obtain a condition ensuring stabilization of (4.6) different from that of Yong. For if the spectral radius of $A$ is less than $N /(N+1)$, we let

$$
K=\left(\varepsilon-\frac{N^{N}}{(N+1)^{N+1}}\right) B^{-1} A^{N+1}
$$

and the asymptotic stability of

$$
x(k+1)=A x(k)+B K x(k-N)
$$

follows.

Ultimately, it is of interest to generalize from the restriction that $B$ is $n \times n$ in (1.2), (1.3). In this regard, an explicit formulation of the stabilizing matrix $K$ becomes difficult. It can be shown [9], however, that controllability of the pair $[A, B]$ with certain restrictions on the spectrum of $A$ implies the controllability of the pair $\left[A_{D}, B_{D}\right]$. So that if we assume $b$ to be $n \times 1$ and $[A, b]$ completely controllable in the continuous system (1.2), the $r$-stabilization of (1.9), the discrete-time version of (1.2), (1.3), follows from results of Yong [13].

These issues will be addressed in a forthcoming paper.

\section{REFERENCES}

[1] E. J. Barbeau, Polynomials, Springer-Verlag, New York, 1989

[2] K. L. Cooke and J. Wiener, Retarded differential equations with piecewise constant delays, J. Math. Anal. Appl. 99, 265-297 (1984)

[3] B. A. Francis and T. T. Georgiou, Stability theory for linear time-invariant plants with periodic digital controllers, IEEE Trans. Autom. Control AC-33, 820-832 (1988)

[4] I. Gyori and G. Ladas, Oscillation theory of delay differential equations with applications, Oxford University Press, 1991

[5] P. T. Kamamba, Control of linear systems using generalized sampled-data hold functions, IEEE Trans. Autom. Control AC-32, 772-783 (1987)

[6] P. Lancaster and M. Tismenetsky, The Theory of Matrices, Academic Press, Ontario, FL, 1985

[7] M. Marden, Geometry of polynomials, Math. Surveys, No. 3, Amer. Math. Soc., Providence, RI, 1966

[8] Q. G. Mohammed, On the zeros of polynomials, Amer. Math. Monthly 72, 631-633 (1965)

[9] E. D. Sontag, Mathematical Control Theory, Springer-Verlag, New York, 1990

[10] J. Wiener and K. L. Cooke, Oscillations in systems of differential equations with piecewise constant argument, J. Math. Anal. Appl. 137, 221-239 (1989)

[11] Y. Yong, Stabilization of linear systems by time-delay feedback controls, Quart. Appl. Math. 45, 377-388 (1987)

[12] J. Yong, Stabilization of linear systems by time-delay feedback controls. II, Quart. Appl. Math. 56, 593-603 (1988)

[13] J. Yong and A. Araposthathis, Stabilization of discrete-time linear systems with a time delay in the feedback loop, Internat. J. Control 48, 1475-1485 (1988) 